\title{
Damping a pendulum's swing by string length adjustment - design and comparison of various control methods*
}

\author{
Milan Anderle ${ }^{1}$, Wim Michiels ${ }^{2}$, Sergej Čelikovský ${ }^{1}$ and Tomáš Vyhlídal ${ }^{3}$
}

\begin{abstract}
A novel nonlinear control theory based feedback controller is proposed to damp the oscillations of the suspended load (pendulum) using the active modification of the length of the suspension string. This setting is a highly nonlinear one since the approximate linearization around the equilibrium working point is neither controllable, nor asymptotically stabilizable. The nonlinear design of the control law is therefore based on the conveniently selected control Lyapunov function. The resulting control law is then compared to the previously developed timedelay feedback control law, both in simulations and using the laboratory experimental realization of the suspended load system. Despite the fact that in the simulations the time-delay feedback control law suppresses the oscillations better than the nonlinear control law, in the experiments the performance of the time-delay feedback and of the nonlinear control law are rather similar. Moreover, the former keeps the pendulum string length oscillating, the latter stabilizes the nominal string length as well. Finally, the numerical optimization shows that the ideal damping would be provided by the impulsive-like control producing piece-wise constant string length dynamics.
\end{abstract}

\section{INTRODUCTION}

The benchmark problem of [1] for the damping of the suspended load oscillations using the adjustment of the suspension rope length is considered here. More specifically, a novel design using the state space model and Lyapunov based nonlinear control techniques is presented and compared with both the previously published solution and another possible alternative, namely the time-delay based approach of [2] and the numerical optimal control approach.

As recognized already in [1], the pendulum's swing suppression is based on its up and down movement synchronized with the swing angle so that the generated Coriolis force acts in the opposite direction to the angular motion, as depicted in Fig. 1. Even though this problem may seem to be rather academic, it may find applications in the crane industry to damp the residual oscillations of the suspended payload when keeping the crane cart at a fixed location. Despite the fact that the way of pendulum swing suppression based on

\footnotetext{
*The first three authors collaborated using the travelling support of the research exchange project VS.060.17N between the Research Foundation Flanders (FWO) and the Academy of Sciences of the Czech Republic. The first and the third author were supported by the Czech Science Foundation through the research grant No. 17-04682S. The fourth author was supported by the Czech Science Foundation through the research grant No. 16-17398S.

${ }^{1}$ The Czech Academy of Sciences, Institute of Information Theory and Automation (ÚTIA AV ČR), 18208 Prague 8, Czech Republic. anderle@utia.cas.cz, celikovs@utia.cas.cz.

${ }^{2}$ Department of Computer Science, KU Leuven, Celestijnenlaan 200A, 3001 Heverlee, Belgium. Wim. Michiels@cs.kuleuven.be

${ }^{3}$ Department of Instrumentation and Control Engineering, Faculty of Mechanical Engineering, and Czech Institute of Informatics, Robotics, and Cybernetics, Czech Technical University in Prague, Czech Republic. tomas.vyhlidalefs.cvut.cz
}

the up and down movement in some aspects overcomes the more common approach based on the cart movement, e.g. by applying the input shaping approach [3], only few references related to this problem can be found. In [1], [4] a harmonic signal for continuous damping of the pendulum's movement was suggested. However, the proposed open loop method required the application of additional signal processing tools to determine the actual value of the oscillation frequency, which slightly varies with the oscillation amplitude of the pendulum. The other task to be solved concerned the synchronization of the up and down motion with the swing motion of the pendulum. Even though this approach was verified in laboratory experiments, see [5] and [6], it is hardly applicable in practice where the regular periodic motion can be affected by additional disturbances (e.g. by forces induced by the wind, in the case of crane application). On the other hand, the closed loop algorithm developed in [2] does not need such an additional signal processing tool as the synchronization is guaranteed directly by the time delay feedback from the pendulum angle's measurement. Robust optimization procedure and extended linearization are employed to attenuate oscillations in the crane systems by the re-configuring the position of the payload mass in [7]. In [8] two energy-based methods are proposed to suppress the pendulum's oscillation by simultaneously controlling the pivot point and weight of the mass of the pendulum. The stabilizing control law that damps the swing of the pendulum having the controllable rod length has been developed using Lyapunov method in [9]. Moreover, in [10] the same authors verified the Lyapunov based approach in a laboratory experiment. A detailed and specific comparison of these results with those of the current paper will be given later on. The analysis of the periodic solutions and their stability properties for the pendulum with the periodically varying length was provided in [11], [12]. Regimes of regular and chaotic motions of the pendulum with the periodically varying length was analyzed in [13].

The current paper provides yet another Lyapunov-based feedback controller which, besides damping the oscillations, also aims to stabilize the pendulum length to its nominal selected designed value. Though it solves a different task that the above mentioned time-delay approach, performance of these approaches is compared both in simulations and real laboratory experiments. Moreover, the open-loop numerical optimization will indicate that the optimal, in a certain sense, damping under limited non-vanishing length variation is given ideally by the impulsively behaving string speed, or, equivalently, by a piece-wise constant string length. 
The rest of the paper is organized as follows. The next section introduces the pendulum with adjustable string and derives its state-space mathematical model, including the Coriolis force effect having a damping potential. Previously known time-delay feedback controller and the novel Lyapunov-based feedback controller are presented in Section III, including their simulation comparison. The optimal openloop control is presented in Section IV, while the experimental validation results are described in Section V. Section VI briefly concludes the paper.

\section{PROBLEM FORMULATION}

The set-up depicted in Fig. 1 will be considered here. Note, that the same set-up was considered in [2] and it will be also used later on for the laboratory experimental verification of the achieved results. Note that the pendulum length is actually adjusted by movable cart via a control force $F(t)$ acting on the suspension string.

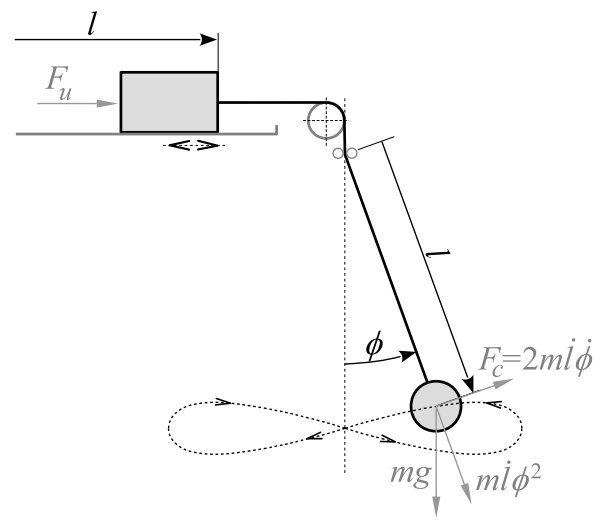

Fig. 1. Scheme of the cart-pendulum system to suspend the oscillations using the Coriolis force.

In order to model this set-up, the ideal pendulum with the adjustable string length is considered. Neglecting the mass of the string, the friction at the pivot, the cart friction and the friction corresponding to the pendulum motion, the governing equation of motion is given by the following nonlinear second order system

$$
m l(t) \ddot{\phi}(t)+2 m \dot{l}(t) \dot{\phi}(t)+m g \sin \phi(t)=0,
$$

where $\phi(t), l(t)$ denote the pendulum angle and length of the string, respectively, $m$ is the mass of the load and $g$ is the gravitational acceleration. The equation (1) describing the motion of the pendulum with variable string length should be completed by the equation describing the dynamics of the string length, namely:

$$
m \ddot{l}(t)=m l(t) \dot{\phi}^{2}(t)+m g \cos \phi(t)-F(t),
$$

where $F(t)$ is the control force to be exerted by a servomechanism to control $l(t)$.

The control design will be performed in two cascades. First, the model where $(\mathrm{d} l / \mathrm{d} t)$ is considered to be "virtual" controlled input will be used, ignoring thereby the equation (2). This simpler model will be used both theoretically and in numerical simulations later on. Further, for the purpose of the laboratory experiments, the above controller is mounted with another simple proportional-derivative (PD) cascade for $F(t)$ that ensures the required properties of $(\mathrm{d} l / \mathrm{d} t)$. Here, preliminary feedback reflecting (2) will be used, namely tuning is made for yet another virtual input $\bar{u}$ defined as

$$
\bar{u}=\ddot{l}(t)=l(t) \dot{\phi}^{2}(t)+g \cos \phi(t)-F(t) / m
$$

and then it is recomputed to the real input $F(t)$ as follows:

$$
F(t)=m l(t) \dot{\phi}^{2}(t)+m g \cos \phi(t)-m \bar{u} .
$$

This helps significantly the tuning of the parameters of the mentioned PD cascade during the laboratory experiments.

\section{PROPOSED FEEDBACK CONTROLLERS}

The possible approaches to design feedback controllers for the previously mentioned "first cascade" are provided here. Namely, $i(t)$ is considered to be a virtual control for the dynamics (1) while ignoring at this stage the string length dynamics (2). First, the control law from [2] is briefly recalled and then the novel Lyapunov based control approach is developed. Subsequently, the behavior of these control laws close to the equilibrium is discussed.

\section{A. Time-delay based control approach}

The control method of [2] generates a harmonic signal of double frequency compared to the pendulum oscillation to be suppressed, according to the current deviation angle of the pendulum. The pendulum length $l(t)$ is considered as the control input, and the control law is expressed as

$$
l(t)=l_{0}-\Delta l_{a} \frac{\phi^{2}\left(t-\tau_{2}\right)-e^{-\xi \pi} \phi^{2}\left(t-\tau_{2}-\tau_{1}\right)}{\phi^{2}\left(t-\tau_{2}\right)+e^{-\xi \pi} \phi^{2}\left(t-\tau_{2}-\tau_{1}\right)},
$$

where $\tau_{1}=\frac{\pi}{2 \Omega}$ and $\tau_{2}=\frac{\pi}{4 \Omega}$. The length adjustment amplitude $\Delta l_{a}$ from the nominal pendulum length $l_{0}$ is given by

$$
\Delta l_{a}=\frac{4}{3} \xi l_{0}
$$

With these parameters, the asymptotic behavior of an ideal second order oscillator with natural oscillation frequency $\Omega$ and damping ration $\xi$,

$$
\ddot{\phi}(t)+2 \xi \Omega \dot{\phi}(t)+\Omega^{2} \phi(t)=0,
$$

is imposed. Note, that the control law (3) was derived by applying trigonometric identities under small angle assumption to the original feed-forward control (proposed in [1]):

$$
l(t)=l_{0}-\Delta l_{a} \sin 2 \Omega t .
$$

\section{B. Lyapunov based control design}

First, introduce the standard state space model of (1) having the following state variables and the controlled input:

$$
x_{1}=\phi(t), x_{2}=\dot{\phi}(t), x_{3}=l(t), u(t)=\dot{l}(t) .
$$

The controlled input $u(t)$ is taken as $u(t)=(\mathrm{d} l / \mathrm{d} t)$. In other words, the system is controlled by the velocity of the string rewinding that can be, indeed, efficiently controlled in many practical settings, as already noted in the Introduction. In 
such a way, the state space model of system (1) takes the following form:

$$
\begin{aligned}
& \dot{x}_{1}=x_{2}, \\
& \dot{x}_{2}=-\frac{1}{x_{3}}\left(2 x_{2} u+g \sin x_{1}\right), \\
& \dot{x}_{3}=u .
\end{aligned}
$$

As a matter of fact, practical considerations require that the state in (8) is restricted to the region

$$
x_{1} \in[-\pi / 2, \pi / 2], \quad x_{2} \in \mathbb{R}, \quad x_{3}>0 .
$$

Indeed, recall that $x_{3}$ is the string length and $x_{1}$ is the angle of the string with respect to a vertical line, so that $\left|x_{1}\right|>\pi / 2$ would cause, at least initially, the free fall of the mass rather than the pendulum like movement.

Further, denote $x=\left(x_{1}, x_{2}, x_{3}\right)^{\top}$. The nominal equilibrium of the state space model $(8)$ is $x^{e q}=\left(0,0, l_{0}\right)^{\top}$, where $l_{0}$ is some desired nominal length of the string. The approximate linearization of (8) around its equilibrium $x^{e q}=\left(0,0, l_{0}\right)^{\top}$ is given by the following pair

$$
\left[\begin{array}{ccc}
0 & 1 & 0 \\
-g / l_{0} & 0 & 0 \\
0 & 0 & 0
\end{array}\right],\left[\begin{array}{l}
0 \\
0 \\
1
\end{array}\right]
$$

which is clearly non-controllable and non-stabilizable. Indeed, it possesses the pair of complex conjugated uncontrollable eigenvalues. Yet, the full nonlinear model will be shown to be asymptotically stabilizable by the smooth state feedback.

The state space approach is natural since the above mentioned practical control goal can be theoretically postulated as the asymptotic stabilization of nonlinear state space model (8) to its equilibrium $\left(0,0, l_{0}\right)^{\top}$. The limitation here is that the asymptotic stabilization approach itself does not provide efficient tools to shape the system's response and to adjust the speed of convergence. Nevertheless, the simulations will show that the Lyapunov state space model based approach provides reasonable results having some advantages with respect to other approaches.

More specifically, the concept used here is the well-known control Lyapunov function (CLF). To prove the asymptotic stability later on, the CLF approach will be combined with LaSalle type conditions for asymptotic stability. An interesting feature here is that the study of the invariance of the set for which the time derivative of the Lyapunov function candidate along the solutions of (8) is zero is rather nontrivial. As a matter of fact, several steps of time differentiation of the relations defining that set along the solutions of (8) are needed.

To proceed with the CLF approach, define first the following Lyapunov function candidate:

$$
V=g x_{3}\left(1-\cos x_{1}\right)+\frac{x_{3}^{2} x_{2}^{2}}{2}+c_{1} \frac{\left(x_{3}-l_{0}\right)^{2}}{2}, \quad c_{1}>0 .
$$

The similar function was used in [9] and [10]. More precisely these papers considered only a two dimensional model together with the first and the second term in (11). These terms are the sum of the kinetic energy and the relative potential energy with respect to fixed $x_{3}$ and the pendulum pointing vertically downwards $\left(x_{1}=0\right)$. The novelty of the current paper consists in considering the length of the string as an additional state variable and complementing the Lyapunovlike function with the "penalty" term $c_{1}\left(x_{3}-l_{0}\right)^{2} / 2$ that will force the system to preserve the prescribed length $l_{0}$. Moreover, the analysis based on the well-known LaSalle principle will provide a proof of asymptotic stability as well. On the contrary, the full state space analysis is missing in [9], [10]. These papers instead discuss some ad hoc way to change $x_{3}(t)$ (denoted there $y(t)$ ), not considering it to be state variable. The invariance issue and LaSalle principle are avoided there by introducing the discontinuous control. Nevertheless, the invariance of the switching surface is not well addressed. Moreover, the discontinuous control of the string velocity is practically unrealistic. The current paper will present a smooth control and a rigorous proof using the LaSalle principle.

The full time derivative of (11) along solutions of (8) is

$$
\begin{gathered}
\dot{V}=g \dot{x}_{3}\left(1-\cos x_{1}\right)+ \\
g x_{3}\left(\sin x_{1}\right) \dot{x}_{1}+x_{3} \dot{x}_{3} x_{2}^{2}+x_{3}^{2} x_{2} \dot{x}_{2}+c_{1}\left(x_{3}-l_{0}\right) \dot{x}_{3},
\end{gathered}
$$

i.e., after the substitution from (8) one has

$$
\begin{gathered}
\dot{V}=g u\left(1-\cos x_{1}\right)+g x_{3}\left(\sin x_{1}\right) x_{2}+ \\
x_{3} u x_{2}^{2}+x_{3}^{2} x_{2}\left(-\frac{1}{x_{3}}\left(2 x_{2} u+g \sin x_{1}\right)\right)+c_{1}\left(x_{3}-l_{0}\right) u \\
\dot{V}=g u\left(1-\cos x_{1}\right)+x_{3} x_{2}^{2} u+ \\
x_{2} x_{3} g \sin x_{1}-2 x_{3} x_{2}^{2} u-x_{3} x_{2} g \sin x_{1}+c_{1}\left(x_{3}-l_{0}\right) u .
\end{gathered}
$$

Summarizing, the final form of $\dot{V}$ is as follows

$$
\dot{V}=\left(g\left(1-\cos x_{1}\right)-x_{3} x_{2}^{2}+c_{1}\left(x_{3}-l_{0}\right)\right) u .
$$

The well-known CLF approach uses a quite natural idea: use the free input variable in (12) to make it at least negative semi-definite. To avoid singularities created by the division the following choice seems to be reasonable:

$$
u(t)=-K\left(g\left(1-\cos x_{1}\right)-x_{3} x_{2}^{2}+c_{1}\left(x_{3}-l_{0}\right)\right) .
$$

Here, $K>0$ is the design parameter that can be tuned, indeed, it will be shown that system (8) is asymptotically stable for any choice of $K>0$. Another design parameter is $c_{1}>0$, present in the definition of the Lyapunov function candidate $V$ in (11). This brings us to the following proposition.

Proposition 3.1: Consider system (8) in the region $[-\pi / 2, \pi / 2] \times \mathbb{R} \times(0, \infty)$. Then $\forall K>0, \forall c_{1}>0$ the control law given by (13) asymptotically stabilizes (8) on any subset of the above region that is invariant with respect to (8) with $u$ given by (13).

Proof: Substituting (13) into (12) one has

$$
\dot{V}=-K\left(g\left(1-\cos x_{1}\right)-x_{3} x_{2}^{2}+c_{1}\left(x_{3}-l_{0}\right)\right)^{2}=\frac{-u^{2}}{K} \text {. }
$$

Equality (14) immediately guarantees Lyapunov stability by virtue of one of the well-known versions of the theorems 
of Lyapunov method. Nevertheless, to investigate asymptotic stability, LaSalle principle has to be engaged as $\dot{V}$ is obviously only negative semi-definite. To proceed with, realize that the set where $\dot{V}=0$ is as follows,

$$
V_{d, 0}=\left\{x \in \mathbb{R}^{3}: g\left(1-\cos x_{1}\right)-x_{3} x_{2}^{2}+c_{1}\left(x_{3}-l_{0}\right)=0\right\} .
$$

To apply LaSalle principle, we need to determine the largest subset of $V_{d, 0}$ which is invariant with respect to system (8). This means that the time derivative of expression

$$
g\left(1-\cos x_{1}\right)-x_{3} x_{2}^{2}+c_{1}\left(x_{3}-l_{0}\right)
$$

along solutions of (8) should also be zero for states belonging to that invariant subset of $V_{d, 0}$. This gives equality

$$
g x_{2} \sin x_{1}-u x_{2}^{2}+2 x_{2} x_{3} \frac{1}{x_{3}}\left(2 x_{2} u+g \sin x_{1}\right)+c_{1} u=0 .
$$

By (14) it holds that $u=0$ for all $x \in V_{d, 0}$ and therefore the last equality can be simplified as

$$
g x_{2} \sin x_{1}+2 g x_{2} \sin x_{1}=0 \quad \Longleftrightarrow \quad x_{2} \sin x_{1}=0 .
$$

Again, for any $x$ belonging to any invariant with respect to (8) subset of $V_{d, 0}$ the time derivative of the expression $x_{2} \sin x_{1}$ along solutions of (8) should be zero, i.e.

$$
\begin{gathered}
0=x_{2}^{2} \cos x_{1}-\sin x_{1}\left(\frac{2 x_{2} u+g \sin x_{1}}{x_{3}}\right)= \\
-\frac{g \sin ^{2} x_{1}}{x_{3}}+x_{2}^{2} \cos x_{1} .
\end{gathered}
$$

Summarizing, for every $x$ belonging to the subset of $V_{d, 0}$, invariant with respect to (8), it should hold that

$$
\begin{aligned}
& g\left(1-\cos x_{1}\right)-x_{3} x_{2}^{2}+c_{1}\left(x_{3}-l_{0}\right)=0, \\
& x_{2} \sin x_{1}=0, \quad \frac{g \sin ^{2} x_{1}}{x_{3}}-x_{2}^{2} \cos x_{1}=0 .
\end{aligned}
$$

Recall, that $x_{1} \in[-\pi / 2, \pi / 2]$ and $x_{3}>0$. The above three equalities then imply that $x_{1}=x_{2}=0, x_{3}=l_{0}$. Indeed, the equality $x_{2} \sin x_{1}=0$ implies that either $x_{2}=0$ or $x_{1}=0$. The last equality of those three equalities and $x_{1}=0$ jointly imply that $x_{2}=0$, the same equality and $x_{2}=0$ imply $x_{1}=0$. In such a way, the last two equalities imply $x_{1}=x_{2}=0$. Substituting $x_{1}=x_{2}=0$ into the first equality gives $x_{3}=l_{0}$.

Summarizing, the set $\left\{x=\left(0,0, l_{0}\right)^{\top}\right\}$ is the only invariant subset of the set $V_{d, 0}$ with respect to (8) and therefore the point $\left(0,0, l_{0}\right)^{\top}$ is the asymptotically stable equilibrium point of (8).

Remark 3.2: The invariant set mentioned in Proposition 3.1 is not so easy to determine theoretically, but it is practically reasonable. Obviously, it can be determined numerically and some limits on the initial velocity $x_{2}^{2}$ would be required not to overshoot the maximal allowed angle $x_{1}$ range $\pm \pi / 2$. Besides, condition $x_{3}>0$ might be easily achieved by taking sufficiently large $c_{1}$ in the length penalty term. Nevertheless, the practical motivation for the crane oscillations suppression reduces the analysis to a theoretical consideration. Indeed, one has to look for control parameters $c_{1}, K$ that ensure much smaller angle $x_{1}$ limits than $\pm \pi / 2$ and, in addition, $x_{3}$ should stay close to its nominal length during the experiments and real applications.

Remark 3.3: The above analysis based on LaSalle's principle is quite interesting also from the tutorial and the educative point of view. Indeed, the majority of the textbook examples show some rather straightforward analysis of the invariance of the set where $\dot{V}=0$. Here, the equation determining that set is quite complex. Moreover, it had to be time differentiated with respect to the system trajectories twice, in order to show that there is only the trivial invariant subset of $V_{d, 0}$.

\section{Discussion}

The linearization of (8) around the equilibrium $\left(x_{1}, x_{2}, x_{2}\right)^{\top}=\left(0,0, l_{0}\right)^{\top}$ with $l_{0}>0$ is not controllable as shown by (10). The novel control law (13) gives the smooth feedback law vanishing at the origin. As a consequence, asymptotic stability is established by the above construction (with in addition a guaranteed region of attraction described by Proposition 3.1 !), yet the asymptotic decay rate is not exponential. On the contrary, control law (3) allows to impose local exponential stability according to a specified damping ratio $\xi$. The price to pay is that the control law is non-smooth at the origin. More precisely, it is scale-invariant, in the sense that the feedback signal is not modified when making the substitution $\phi(t) \leftarrow \alpha \phi(t)$ for any $\alpha \neq 0$. As a consequence the control 'effort' does not reduce when attaining convergence, as will be illustrated in the next section. This endangers the stability when the oscillation amplitude gets close to the measurement noise magnitude. Thus, the length adjustment needs to be stopped before this situation happens, as widely discussed in [2]. In such a way, approach presented in [2] solves the different theoretical setting: rather than the overall asymptotic stabilization of $x$ it provides the output regulation for the output $\left(x_{1}, x_{2}\right)^{\top}$ while the hidden dynamics of both $x_{3}$ and $u$ remains nonvanishing.

\section{Simulation}

The simulation comparison of the nonlinear Lyapunov based controller and the time-delay one is provided here. In both cases the suppression of the pendulum swing with the initial deviation $\phi(0)=\frac{\pi}{2} \mathrm{rad}$ and the zero angular velocity $\dot{\phi}(0)=0$ was simulated. The initial length of the pendulum is $l=l_{0}=1 \mathrm{~m}$. In Fig. 2 one can see the trajectories of the controlled pendulum whereas in Fig. 3 one can see the pendulum length during the vibration suppression.

The behavior of the trajectories reflects the theory presented in the previous section. The pendulum swinging seems not to vanish completely using the nonlinear control (the asymptotic slow decay is related to the lack of local exponential stability). The suppression efficiency of the approach based on the time-delay control law is attributed to the constant amplitude of the control signal despite the decaying amplitude of the oscillation to be suppressed. Finally, trajectories of the load are depicted in Fig. 4. 


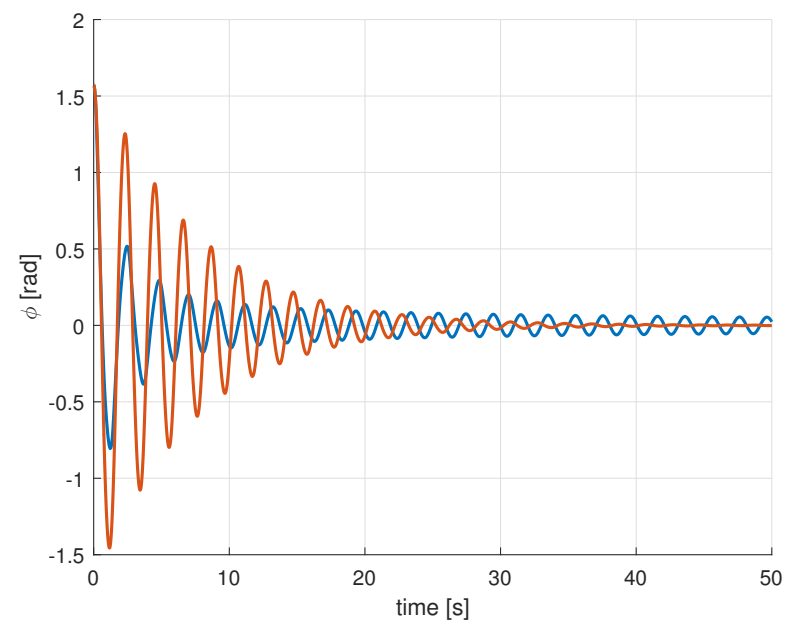

Fig. 2. Simulations: the time evolution of the pendulum angle during the controlled swing. The blue line corresponds to (13), the red line to (3).

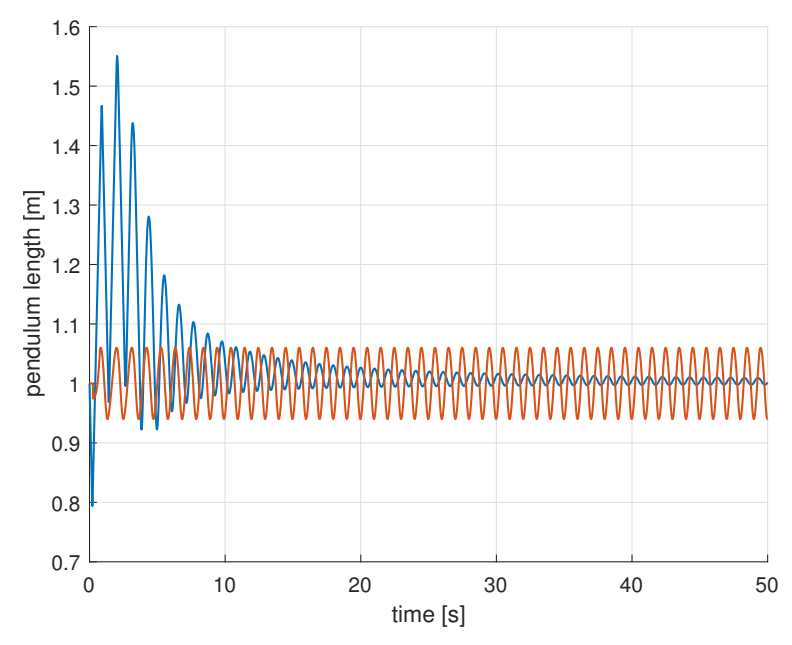

Fig. 3. Simulations: the time evolution of the pendulum length during the controlled swing. The blue line corresponds to (13), the red line to (3).

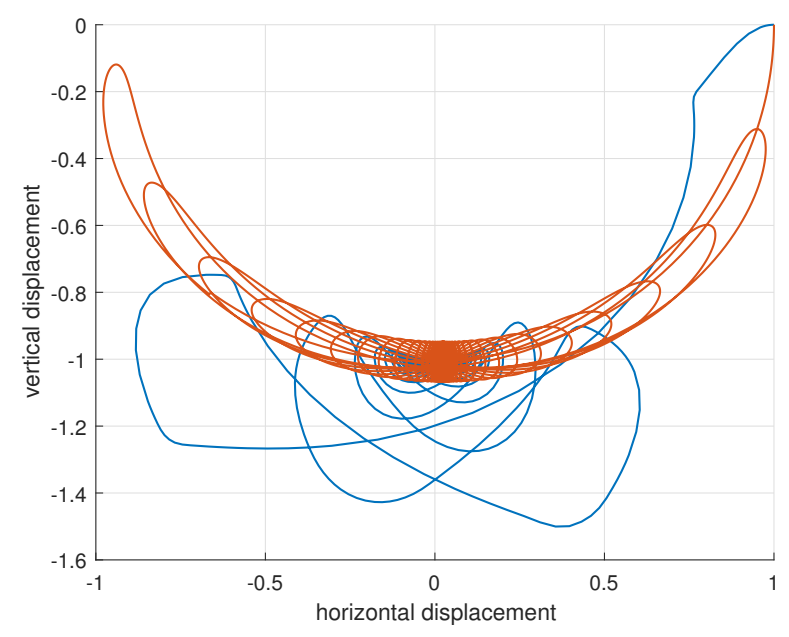

Fig. 4. Simulations: the Carthesian trajectory of the mass. The blue line corresponds to (13), the red line to (3).

\section{OPTIMAL OPEN-LOOP CONTROL}

As an alternative to the Lyapunov based design, we provide the approach based on optimal control theory. The results of computing numerically the open-loop optimal control for the properly defined performance index are presented. They will help to further compare the time-delay and Lyapunov based approaches. Note, that these results concern open loop control, which cannot be directly implemented on the setup (unless implicit feedback is introduced by recomputing the control inputs online over a receding horizon, i.e. by adopting the model predictive control setting).

The nominal length $l_{0}=1$ and the horizon $T=20$ is selected. Then, the minimization of the performance index

$$
J(u)=\int_{0}^{T}\left(x_{1}^{2}(t)+x_{2}^{2}(t)\right) d t+\rho\left(x_{1}^{2}(T)+x_{2}^{2}(T)\right)
$$

with $\rho=8 / 3$, subject to the constraint

$$
\left|x_{3}-l_{0}\right| \leq 0.15,
$$

is performed. Note, that the last constraint actually indicates that the numerical optimal control approach solves very similar problem as the time-delay one, namely, it allows the string length not to vanish.

A discretize-first approach is taken next. States and controls are discretized with time-step $h=2 / 30$ and the cost function is discretized accordingly. Runge-Kutta method of order four is used to approximate the dynamics described by (8). These are treated as constraints in the nonlinear programming problem (NLP), with variables being the discretized states and controls (simultaneous approach). The NLP was solved by MATLAB function fmincon, to which derivatives of cost function and constraints, obtained using automatic differentiation (AD), were provided.

The trajectories corresponding to the optimal control in the interval $[0,10]$ are plotted in Figures 5-6.

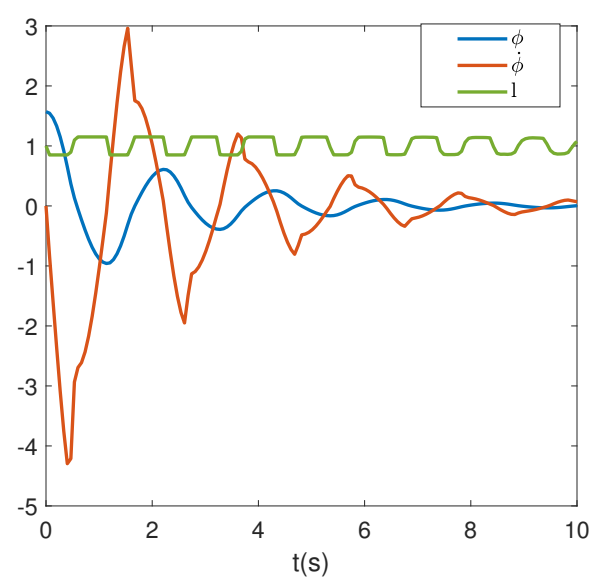

Fig. 5. Simulations: the time evolution of the pendulum's angle (blue), the angular velocity (red) and the string length (green) - optimal control.

The non-vanishing nature of the control compensates the non-controllability of the linearization at the equilibrium to be reached. This is similar to the time-delay based control approach of Section III-A. Yet, the optimal control 


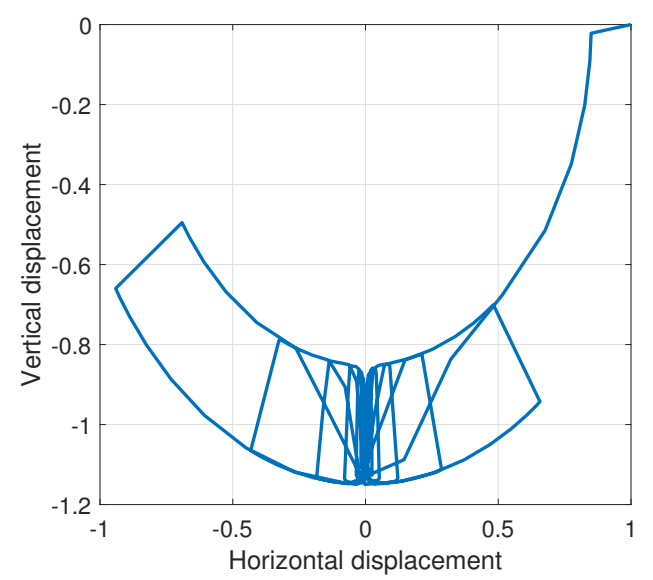

Fig. 6. Simulations: the Carthesian trajectory of the mass - optimal control.

approximates a bang-bang control, with the length (variable $l=x_{3}$ ) quickly changing between extreme values, and as a consequence, a close to impulsive input signal $(u=\dot{l})$. Note, that the sinusoidal like length resulting from the application of (3) can be seen as an approximation of a block wave in bang-bang control.

Moreover, the results shown in Figs. 5, 6 are even more close to those presented in Fig. 8 of [1] that are based on estimating the damping ratio during the saw-tooth-like approximation of the bang-bang length variation. In such a way, the numerical results of the current section show that the strategy developed in [1] is close to the optimal one in the sense of (15).

\section{EXPERIMENTAL VALIDATION OF THE LYAPUNOV-BASED FEEDBACK CONTROLLER}

The newly developed nonlinear feedback control law based on the control Lyapunov function was verified using the experimental setup depicted in Fig. 7. Moreover, the damping effect of the nonlinear feedback control law was compared with the previously developed time-delay based feedback control law.

The experimental setup proposed in [2] consists of an externally controlled movable cart connected with the suspended load via a string of fixed length. As it can be seen in the detail in Fig. 7, the string passes through a pulley of fixed position and an arm of the rotational sensor measures the pendulum angle. The length of the pendulum string is controlled via the position control of the movable cart, which is measured by the incremental sensor.

For the case of the time-delay feedback control law represented by (3) the input of the controller is the angular position $\phi$, and the output is the instantaneous required length of the pendulum $l$. To implement it, the position PD controller was designed and used to adjust the desired pendulum length via the position control of the cart. However, in the case of the nonlinear control law (13) its output $u(t)$ represents the desired velocity of the pendulum string $i$ (in accordance with (8)). For this purpose, a linear velocity observer was

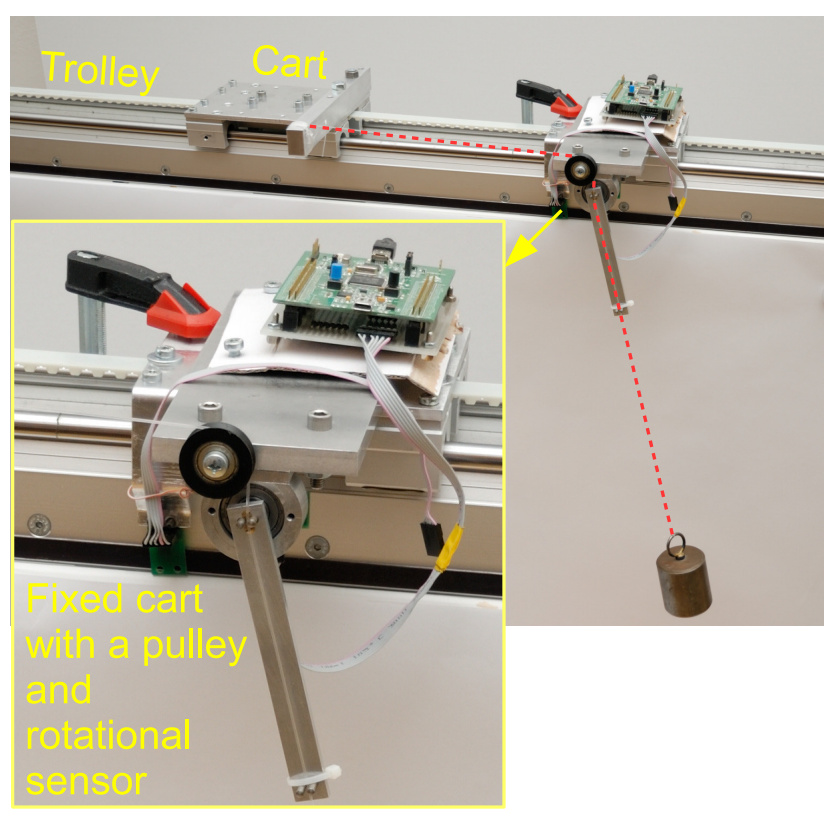

Fig. 7. The experimental setup proposed in [2]

developed and the velocity PD controller was designed to adjust the instantaneous desired velocity of the pendulum string via the velocity control of the cart.

In Fig. 8 one can see behavior of the fixed length (uncontroled) pendulum swing, which is only slightly damped. Results for the Lyapunov-based nonlinear control law are shown in Fig. 9, in comparison with the time-delay feedback control. The corresponding length adjustment of the string is shown in Fig. 10 for both cases. It is clear that the time-delay control approach suppresses the pendulum swing from $t=3.6 \mathrm{~s}$ to $t=13 \mathrm{~s}$, but then it had to be turned off for the reasons discussed above. On the contrary the nonlinear control approach suppressed the pendulum swing from $t=4.2 \mathrm{~s}$ to $t=20 \mathrm{~s}$ when it was turned off. However, after $t=14 \mathrm{~s}$ its damping performance is very small.

It is clear that both pendulum swing suppression approaches work very well. In comparison to the undamped pendulum swing depicted in Fig. 8, considerably higher damping of the swing was achieved. An interesting point is the fact that both approaches suppress the pendulum swing in a very similar way, in contrast to the simulations. Indeed, in Fig. 9 one can see that with almost the same initial deviation of the pendulum around 1.2 rad the pendulum swing was suppressed to around $\pm 0.1 \mathrm{rad}$ within nearly $20 \mathrm{sec}$ with both approaches.

The only significant difference between the approaches is in the evolution of the length adjustment shown in Fig. 10. With the time-delay based control approach, the length oscillates around the predefined initial length of the pendulum. For the Lyapunov based control approach, the length is significantly extended in the first phase. After the swing angle is reduced, it is shortened back to the initial length. The significant extension of the pendulum length could result in a certain disadvantage of the Lyapunov based control approach 
in practice. On the other hand, the time-delay based control algorithm must be switched off when the pendulum swing is suppressed into a certain range, otherwise the constant up and down movement of the pendulum would excite vibrations of the load instead of suppressing them. In contrast to this, the Lyapunov based control approach decreases the control effort as the pendulum swing decreases.

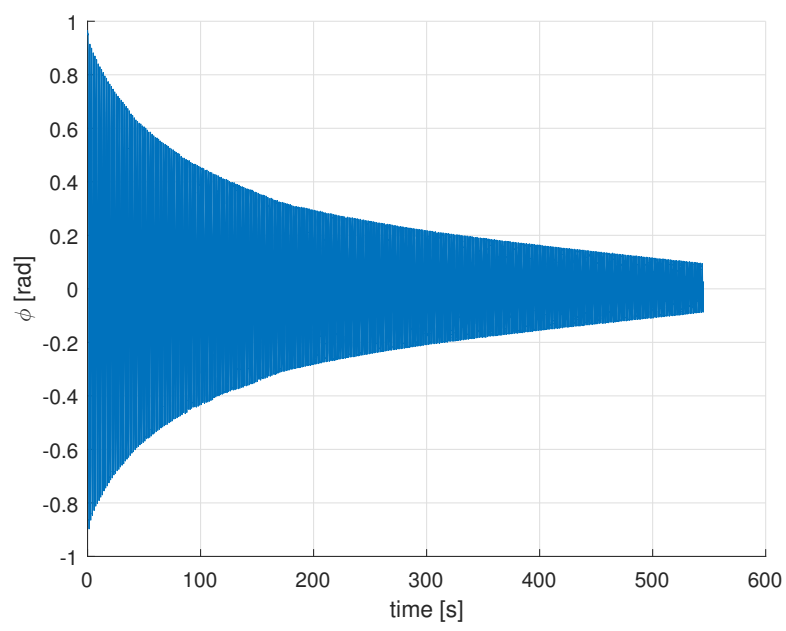

Fig. 8. Experiments: the time evolution of the pendulun angle during the uncontrolled swing.

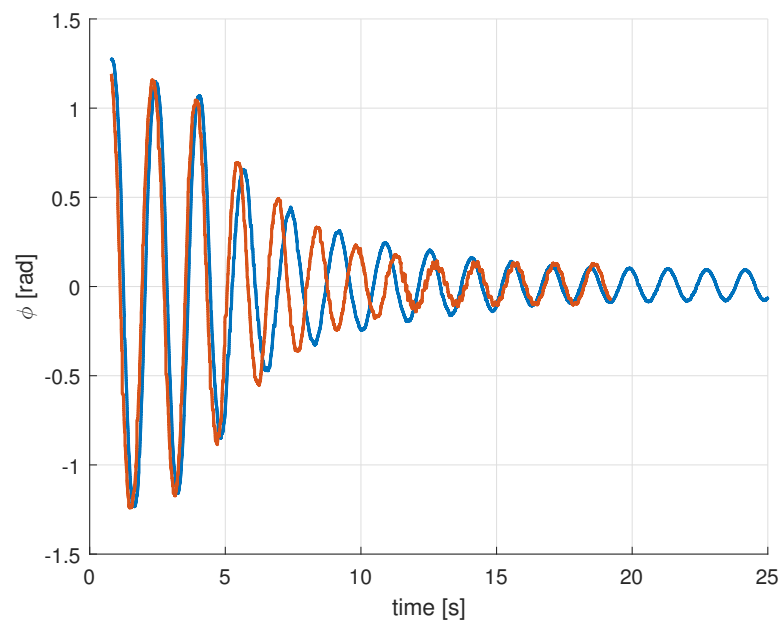

Fig. 9. Experiments: the time evolution of the pendulum angle during the controlled swing. The blue line corresponds to (13), the red line to (3).

\section{CONCLUSIONS}

A novel nonlinear feedback control law based on the control Lyapunov function approach was proposed for the problem of the oscillation suppression of the suspended load by adjusting the length of the suspension string. The suppression performance of the nonlinear control law was compared with the previously known time-delay feedback control law in simulations as well as using the experimental setup. Moreover, the performance of the closed-loop systems was also assessed in relation to the numerically computed solutions of the optimal control problem for the properly defined performance index.

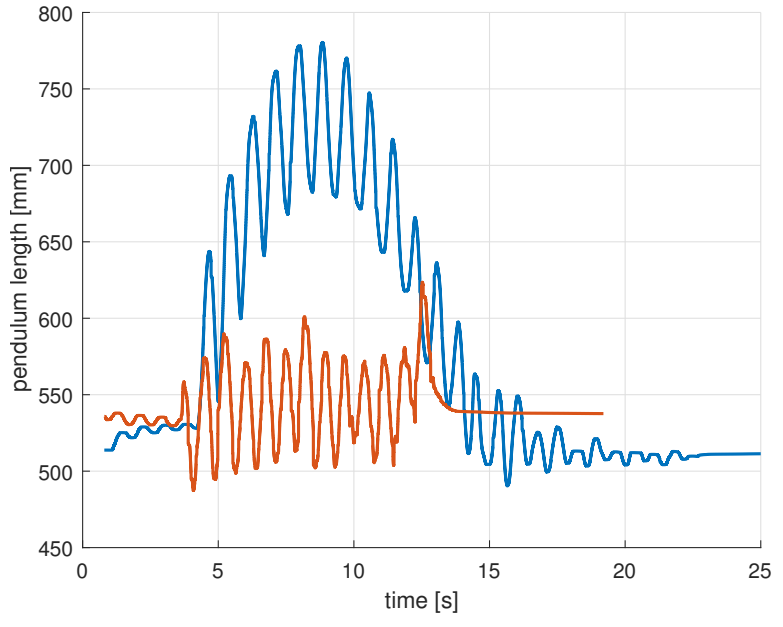

Fig. 10. Experiments: the time evolution of the pendulum length during the controlled swing. The blue line corresponds to (13), the red line to (3).

\section{REFERENCES}

[1] D. Stilling and W. Szyszkowski, "Controlling angular oscillations through mass reconfiguration: a variable length pendulum case," International Journal of Non-Linear Mechanics, vol. 37, no. 1, pp. $89-99,2002$.

[2] T. Vyhlídal, M. Anderle, J. Bušek, and S. Niculescu, "Time-delay algorithms for damping oscillations of suspended payload by adjusting the cable length," IEEE/ASME Transactions on Mechatronics, vol. 22, no. 5, pp. 2319-2329, 2017.

[3] W. Singhose, "Command shaping for flexible systems: A review of the first 50 years," International Journal of Precision Engineering and Manufacturing, vol. 10, no. 4, pp. 153-168, 2009.

[4] W. Szyszkowski and D. Stilling, "On damping properties of a frictionless physical pendulum with a moving mass," International Journal of Non-Linear Mechanics, vol. 40, no. 5, pp. 669 - 681, 2005.

[5] S. Okanouchi, K. Yoshida, I. Matsumoto, and H. Kawabe, "Damping angular oscillations of a pendulum under state constraints," IFAC Proceedings Volumes, vol. 41, no. 2, pp. 7735-7742, 2008.

[6] A. Bellino, A. Fasana, E. Gandino, L. Garibaldi, and S. Marchesiello, "A time-varying inertia pendulum: analytical modelling and experimental identification," Mechanical Systems and Signal Processing, vol. 47, no. 1, pp. 120-138, 2014.

[7] A. Rauh, R. Prabel, and H. Aschemann, "Oscillation attenuation for crane payloads by controlling the rope length using extended linearization techniques," in 2017 22nd International Conference on Methods and Models in Automation and Robotics (MMAR), 2017, pp. 307-312.

[8] K. Yoshida, S. Okanouchi, and H. Kawabe, "Vibration suppression control for a variable length pendulum with a pivot movable in a restricted range," in 2006 SICE-ICASE International Joint Conference, 2006, pp. 4538-4544.

[9] K. Yoshida, H. Kawabe, and K. Kawanishi, "Stabilizing control for a single pendulum by moving the center of gravity. an investigation by numerical experiment," in Proceedings of 35th IEEE Conference on Decision and Control, vol. 1, 1996, pp. 1039-1040 vol.1.

[10] K. Yoshida, I. Kawanishi, and H. Kawabe, "Stabilizing control for a single pendulum by moving the center of gravity: theory and experiment," in Proceedings of the 1997 American Control Conference (Cat. No.97CH36041), vol. 5, 1997, pp. 3405-3410 vol.5.

[11] A. Pinsky and A. Zevin, "Oscillations of a pendulum with a periodically varying length and a model of swing," International Journal of Non-Linear Mechanics, vol. 34, no. 1, pp. 105 - 109, 1999.

[12] A. Zevin and L. Filonenko, "Qualitative study of oscillations of a pendulum with periodically varying length and a mathematical model of swing," Prikl. Mat. Mekh., vol. 71, pp. 989-1003, 012007.

[13] A. Belyakov, A. Seyranian, and A. Luongo, "Dynamics of the pendulum with periodically varying length," Physica D: Nonlinear Phenomena, vol. 238, no. 16, pp. 1589 - 1597, 2009. 